\title{
Novelty and temporal contiguity in taste aversion learning: Within-subjects conditioning effects
}

\author{
JOSEPH J. FRANCHINA, SARA SILBER, and BRIAN MAY \\ Virginia Polytechnic Institute and State University, Blacksburg, Virginia 24061
}

\begin{abstract}
Rats $(\mathrm{N}=144)$ received preexposure to $12 \%$ sucrose, $.9 \%$ saline, or tap water prior to aversion training in which they all received presentation of saline and sucrose in series before an intraperitoneal injection of .15-M lithium chloride. Between-groups comparisons of aversion effects, measured with a two-bottle choice procedure (i.e., sucrose or saline vs. tap water), showed that preexposure to sucrose or to saline eradicated aversion to the familiarized flavor but did not affect aversion to the novel flavor. Aversion effects to the novel flavor following sucrose or saline preexposure were highly similar to those that occurred to novel sucrose and saline flavors following tap water preexposure. Order of flavor presentation in aversion training did not affect the reliable demonstration of differential aversion effects to novel and familiarized taste cues.
\end{abstract}

A consistent finding in taste aversion research is that preexposure to a novel flavor prior to pairing that flavor with toxicosis reliably reduces the magnitude of aversion effects (e.g., Elkins, 1973; Fenwick, Mikulka, \& Klein, 1975; Kalat, 1974). In studying the effects of flavor novelty within subjects, Ahlers and Best (1971) and Revusky and Bedarf (1967) reported that serial presentation of a familiar and an unfamiliar conditioning flavor prior to toxicosis yielded greater aversion to the novel flavor for the poisoned group than for nonpoisoned controls. Further, aversion effects were unrelated to the temporal contiguity between flavor presentation and toxicosis: Regardless of the immediateness with which the familiarized or the novel flavor preceded the toxic agent, aversion effects were greater to the novel flavor. These data suggested that taste aversion learning may depend more on flavor novelty than on the temporal contiguity between taste cues and toxicosis.

In testing for aversion effects, Ahlers and Best (1971) and Revusky and Bedarf (1967) used a two-bottle choice procedure that provided simultaneous access to the familiarized and the novel flavors. Aversion to the novel flavor was measured by intake of that flavor relative to the combined intakes of the novel and the familiarized flavors. However, the inclusion of both flavor conditions in testing and in the measure of aversion to the novel flavor raises the possibility that responding to one of the flavor conditions was affected by the presence of the other. If so, then it becomes difficult to assess the separate contributions of the novel and familiarized

These data were presented at the meeting of the Southeastern Psychological Association, Washington, D.C., March 1980. Requests for reprints should be sent to J. J. Franchina, Department of Psychology, V.P.I. \& S.U., Blacksburg, Virginia 24061. The authors thank B. Benson for critically reading this paper. flavor conditions to the magnitude of the aversion effect obtained. Further, if aversion performance reflected a contrast/comparison between flavor conditions, the basis of this comparison may have been original training, aversion testing, or both.

Revusky and Bedarf (1967) also reported that in within-subjects testing, flavor preference apparently influenced the demonstration of differential aversion effects. When the pair of flavors in testing was sucrose and milk, aversion effects to the novel flavor were reliable when the novel flavor was sucrose (milk being the familiarized flavor), but not when it was milk. When milk and grape juice composed the flavor pair in testing, aversion was reliable when the novel flavor was milk, but not when it was grape juice. Thus, these data suggested that in within-subjects comparisons of familiar vs. novel flavors, flavor preference effects may contaminate the demonstration of flavor novelty effects.

The purpose of the present experiment was to study within-subjects effects of flavor novelty and flavortoxicosis contiguity under conditions that minimized the complications of earlier reports. To separate the effects of within-subjects conditions of training from those of testing, all rats were first administered aversion training with within-subjects presentations of a novel and a familiarized conditioning flavor. Then, independent groups were tested for aversion effects with a twobottle choice procedure that provided access to one of the two conditioning flavors and tap water. Betweengroups comparisons of test-trial performance should indicate the extent of within-subjects conditioning effects. Specifically, rats received pretraining access to $12 \%$ sucrose, $.9 \%$ saline, or tap water. Subsequently, all rats received access to sucrose and to saline in a counterbalanced order, followed by an injection of $\mathrm{LiCl}$. In aversion testing, independent groups received 
a two-bottle choice. One bottle contained sucrose or saline; the other contained tap water.

\section{METHOD}

\section{Subjects}

Subjects were 144 experimentally naive albino rats (Rattus Norvegicus), 72 male and 72 female Sprague-Dawley descendents, bred in the animal colony of the Department of Psychology at Virginia Polytechnic Institute and State University. The rats were 86-91 days old on Day 1 of the experiment.

\section{Procedures}

Throughout the experiment, each rat was housed in a singlehanging cage in a room $2.4 \times 2.4 \times 3.0 \mathrm{~m}$. Room temperature was $78^{\circ} \pm 2^{\circ} \mathrm{F}$; illumination was approximately $80 \mathrm{fc}$ on a 10-h/14-h light/dark cycle. Light onset was at $0800 \mathrm{~h}$; offset was at $1800 \mathrm{~h}$. Rat chow and tap water were continuously available except as noted.

The experiment lasted 13 days. On Day 1 , each rat was caged and received access to chow and tap water for the first $12 \mathrm{~h}$. Water access was then discontinued for $12 \mathrm{~h}$ to initiate deprivation procedures. On Day 2, rats received $1 \mathrm{~h}$ access to tap water beginning at 0800,1400 , and $2000 \mathrm{~h}$.

On the morning of Day 3, the rats were randomly assigned to cells $(n=12)$ of a 3 by 2 by 2 design that combined three preexposure fluids $(12 \%$ sucrose $w / v, .9 \%$ saline w/v, or tap water) with two injection conditions (immediate or delayed $\mathrm{LiCl}$ injections) and two test flavors (sucrose or saline). For fluid preexposure, rats $(n=48)$ received two 30 -min periods of access to $12 \%$ sucrose, $.9 \%$ saline, or tap water, starting at approximately 0830 and $2030 \mathrm{~h}$, respectively, on Days 3 and 4 .

On Day 5, each rat received the following sequence of aversion training: $2 \mathrm{~min}$ access to either sucrose or saline, $10 \mathrm{~min}$ without any fluids, and $2 \mathrm{~min}$ access to the other flavor of the conditioning pair (saline or sucrose). After the end of the second access period, all rats were injected intraperitoneally with 3.0 milliequivalents of .15-M LiCl. Rats in the immediate- $\mathrm{LiCl}$ condition ( $\mathrm{LiCl})$ were injected $10 \mathrm{~min}$ afterward; rats in the delayed- $\mathrm{LiCl}$ condition (DeLi) were injected $12 \mathrm{~h}$ later. In training, sucrose and saline presentations were counterbalanced within each cell of the experimental design. Sucrose access preceded saline access for one half of the rats and followed saline access for the other half. On Day 6, the recovery day, all rats received $15 \mathrm{~min}$ access to tap water at approximately $0830 \mathrm{~h}$ and $30 \mathrm{~min}$ access at approximately $2030 \mathrm{~h}$.

Aversion test trials occurred on Days 7-13, at 1 trial/day. A daily trial consisted of $15 \mathrm{~min}$ access to a two-bottle choice situation. One bottle contained a conditioning flavor, either sucrose or saline, depending on the rat's group assignment; the other bottle contained tap water. Thus, one half of the rats were tested with sucrose vs. tap water and the other half with saline vs. tap water. Eight hours after a test trial $(1600 \mathrm{~h})$, all rats received $30 \mathrm{~min}$ access to tap water. Food was continuously available ad lib throughout the experiment, except during flavor access periods of preexposure, training, and testing. At those times, food was removed from the home cage $30 \mathrm{~min}$ before the period started and was returned $6 \mathrm{~h}$ later.

The measure of performance was the amount of fluid ingested, as calculated (within $.1 \mathrm{ml}$ ) by the difference in a bottle's weight from the beginning to the end of an access period. In aversion testing, a preference index was obtained for each flavor on each test trial: namely, test flavor intake relative to total fluid intake (test flavor plus tap water intake). For sucrose, this index was Suc/(Suc $+\mathrm{HOH}) \times 100$.

Sucrose and saline solutions were mixed $18 \mathrm{~h}$ prior to the start of each access period. Sucrose was mixed with commericalgrade sugar and distilled water. Saline was mixed with reagentgrade sodium chloride (Fisher Company) and distilled water.

\section{RESULTS}

Results for Preexposure Periods 1-4 showed that sucrose intake (in milliliters) generally exceeded saline and tap water intakes (grand means $=11.6,9.8$, and 8.4, respectively). However, in Period 4, sucrose and saline intakes were highly similar to each other (means $=12.8$ and 13.0) and were greater than tap water intake (mean = 8.7). Analysis of variance of all the data of preexposures yielded reliable effects for fluid condition, preexposure periods, and Fluid Conditions by Preexposure Periods $[F(2,141), F(3,423)$, and $F(6,423)=15.32,27.54$, and 14.11 , respectively; ps $<.001]$.

Figure 1 shows mean flavor preference over all test trials for familiarized $(\mathrm{F})$ and novel $(\mathrm{N})$ conditions of sucrose and saline test flavors following preexposure to saline (Na), sucrose (Su), or tap water (TW). Results are shown for F-N, N-F and Na-Su, Su-Na orders of flavor presentation in training under immediate $(\mathrm{LiCl})$ or 12-h-delayed (DeLi) injections of $\mathrm{LiCl}$.

Comparisons between F-N and N-F training sequences provide information on the relative influence of temporal contiguity and flavor novelty in aversion learning. In general, the effects of F-N and N-F sequences were similar to each other under familiar and under novel test conditions following sucrose and saline preexposures in both injection conditions. In the familiarized flavor test condition, mean preference was approximately the same whether the familiarized flavor preceded (F-N) or followed (N-F) the novel flavor; in the novel flavor test condition, mean preference was also nondifferential across F-N and N-F training flavor sequences. Comparisons between $\mathrm{LiCl}$ and $\mathrm{DeLi}$ sections of Figure 1 further showed that F-N and N-F training sequences produced magnitudes of taste aversion essentially similar in familiar and in novel test conditions for saline and for sucrose preexposed groups. Analysis of variance of the data for F-N and N-F conditions in Figure 1 revealed no reliable main effect nor interactions for F-N/N-F order of flavor presentation in aversion training ( $F s<1)$. Together with the data of Figure 1, these results indicated that aversion effects toward novel and familiarized flavors developed independently of the contiguity between flavor presentation (within subjects) and toxicosis. Interestingly, when saline and sucrose were both novel conditioning flavors (i.e., following tap water preexposure), preference and aversion effects were also independent of the order of flavor presentation in training. Sucrose preference exceeded saline preference in $\mathrm{LiCl}$ and $\mathrm{DeLi}$ conditions, regardless of the temporal proximity between these flavors and toxicosis.

Figure 2 presents mean flavor preference for sucrose (top graph) and for saline (bottom graph) on. Test Trials 1-7 in $\mathrm{LiCl}$ and $\mathrm{DeLi}$ conditions following sucrose, saline, or tap water preexposure. The data for F-N and $\mathrm{N}-\mathrm{F}$ training flavor sequences were combined for each group in this figure. 


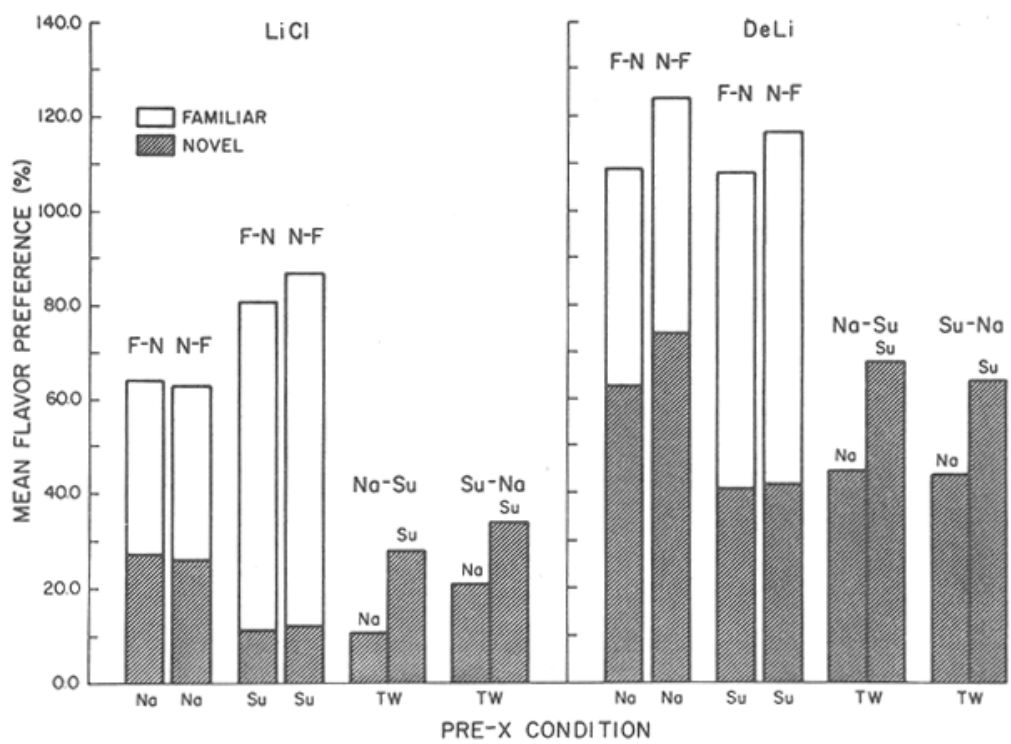

Figure 1. Mean flavor preference in testing with familiar (F) and novel (N) conditions of sucrose and saline for groups administered immediate $(\mathrm{LiCl})$ or 12-h-delayed (DeLi) injection of lithium chloride in aversion training following saline (Na), sucrose (Su), or tap water (TW) preexposure (Pre-X). Order of flavor presentations in aversion training was familiar-novel (F-N) or novel-familiar (N-F) for saline or sucrose preexposed groups and saline-sucrose ( $\mathrm{Na}-\mathrm{Su})$ and sucrose-saline (Su-Na) for tap water preexposed groups.

In sucrose (top graph) and in saline (bottom graph) testing, preexposure to the test flavor precluded the demonstration of aversion to that flavor but did not affect aversion to the novel flavor of each conditioning pair. Specifically, following sucrose preexposure, the difference between $\mathrm{LiCl}$ and $\mathrm{DeLi}$ groups was negligible for the familiarized sucrose test flavor (top graph) but was larger for the unfamiliarized saline test flavor (bottom graph). Following saline preexposure, the $\mathrm{LiCl}-\mathrm{DeLi}$ difference was negligible for the familiarized saline test flavor but was larger for the unfamiliarized sucrose test flavor. In each test condition, the level of aversion to the novel flavor of the conditioning pair following flavor preexposure was highly similar to that shown for sucrose and saline following tap water preexposure. Finally, aversion effects were generally larger to novel sucrose than to novel saline; this finding may reflect a generally greater preference for sucrose, as results of $\mathrm{DeLi}$ groups suggest.

Analysis of variance of all the data of Figure 2 yielded reliable effects for each main factor, fluid preexposure, test flavor, injection condition, and test trials $(\mathrm{p}<.05)$, but,. more important, for the interactions, Fluid Preexposure by Injection Condition by Test Flavor $[F(2,132=23.40, p<.001]$ and Injection Condition by Test Flavor by Test Trials $[F(6,792=$ $3.46, p<.002]$. Simple-effects analyses of variance for each test condition showed that in sucrose testing, the

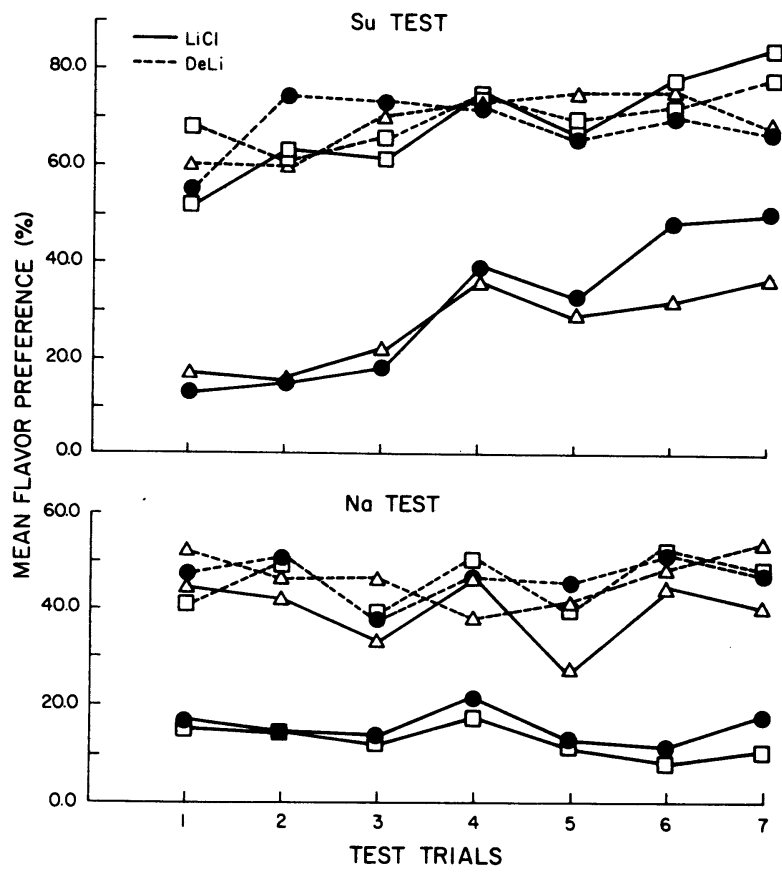

Figure 2. Mean flavor preference on Test Trials 1-7 for groups tested with sucrose (top graph) or saline (bottom graph) following preexposure to sucrose (squares), saline (triangles), or tap water (closed circles) and aversion training with immediate (LiCl) or 12-h-delayed (DeLi) injections of lithium chloride. 
difference between $\mathrm{LiCl}$ and $\mathrm{DeLi}$ groups was reliable for saline and for tap water preexposed groups $(\mathrm{p}<.001)$, but not for the sucrose preexposed group $(\mathrm{F}<1)$. For saline testing, the $\mathrm{LiCl}-\mathrm{DeLi}$ difference was reliable for sucrose $(p<.003)$ and for tap water $(p<.005)$ preexposed groups, but not for the saline group $(\mathrm{F}<1)$. It should be noted that for tap water groups, aversion effects were reliably established to both conditioning flavors, sucrose and saline, whose novelty status was presumably unchanged by tap water preexposure. Finally, an analysis of variance over the data of $\mathrm{DeLi}$ groups alone for both test conditions yielded a reliable effect only for test flavor $[\mathrm{F}(1,66)=63.69, \mathrm{p}<.001]$. This finding suggests that the difference in aversion magnitude between sucrose and saline testing in Figure 2 may reflect, in part, a difference in preference between sucrose and saline flavors.

\section{DISCUSSION}

This experiment reliably demonstrated differential aversion effects to novel and familiarized conditioning flavors, independent of the order of flavor presentation (within subjects) prior to toxicosis. Aversion effects were reliably greater to the novel flavor of each conditioning pair; aversion to the novel flavor was greater in the poison conditioning groups ( $\mathrm{LiCl})$ than in the poison control group (DeLi). Thus, these results supported data from Ahlers and Best (1971) and Revusky and Bedarf (1967), showing that, in aversion learning, relative flavor novelty was more important than temporal contiguity between flavor and toxicosis.

Contrary to earlier procedures (e.g., Ahlers \& Best, 1971; Revusky \& Bedarf, 1967), the present experiment used betweengroups comparisons of test-trial performance to show differential aversion effects following within-subjects training. As a result, it appears that the finding of within-subjects effects of flavor novelty does not require the simultaneous presence of familiar and novel flavors in testing. Rather, the present data seem to constitute evidence for differences in the original conditioning of aversion to familiar and novel flavors during training. Preexposure to a novel flavor presumably reduces flavor novelty and, thereby, curtails the conditionability of the preexposed flavor in later aversion training. Within-subjects presentation of preexposed and nonpreexposed flavors presumably permits a differentiation between the flavors on a historical basis and in terms of their associability with toxicosis. Kalat (1974) and Kalat and Rozin (1970) indicated that flavor novelty regulates associability with toxicosis. In the present experiment, then, the relatively more novel flavor of each conditioning pair would be the more associable with toxicosis and differential aversion learning should occur as it seemed to.

Flavor novelty describes a relative state in the organism's history. Thus, it is possible that aversion learning to the novel flavor was affected by the availability of the familiar flavor in original training. Presentation of the latter might have accentuated the newness (unfamiliarity) of the novel flavor, thereby increasing that flavor's associability with toxicosis and enhancing the level of aversion conditioning beyond that conditionable to the novel flavor per se. Within-subjects conditioning procedures seem to promote evidence for relational learning (e.g., Grice, 1968). In this experiment, however, aversion learning seems to have been specific to the novelty status and the quality of the flavor stimuli. Following flavor preexposure, aversion effects to the novel flavor of each $\mathrm{F}-\mathrm{N}$ conditioning pair did not differ reliably from that conditioned to novel sucrose or saline flavors following preexposure to tap water. Tap water preexposure presumably did not alter the novelty status of sucrose or saline. Thus, the conditioning effects subsequently obtained should have indicated the flavors' associability with toxicosis under withinsubjects procedures. The lack of reliable aversion differences between novel conditioning flavors following tap water preexposure and those novel flavors following sucrose/saline preexposure suggests that, in the latter case, the presence of the familiarized flavor did not appreciably affect aversion learning to the novel flavor. In addition, the finding of similar aversion effects suggests that for flavor preexposure groups, pretraining experience with one novel flavor apparently did not affect aversion learning to a second novel flavor. These data are consistent with results from Braveman and Jarvis (1978).

Finally, comparisons between $\mathrm{LiCl}$ and $\mathrm{DeLi}$ conditions showed that aversion effects were greater to novel sucrose than to novel saline. This difference was not reflective of performance in the poison conditioning group $(\mathrm{LiCl})$ but seemed to be due to the reliably greater preference for sucrose over saline in the poison control groups (DeLi). If the DeLi group's data indicated the relative acceptability of sucrose and saline, then they seem to vindicate the use of between-groups comparisons of test performance in the present experiment. If both conditioning flavors had been simultaneously presented in within-subjects test procedures, it is likely that flavor preference effects could have contaminated flavor novelty effects, as Revusky and Bedarf (1967) suggested in their report. Aversion might have occurred to the novel flavor of the testing pair when the relatively less preferred flavor occupied the novel position, but not when the more preferred flavor was the novel designate. Use of between-groups comparisons in testing circumvented this potential problem in the present experiment, substantiated the conclusions of Ahlers and Best (1971) and Revusky and Bedarf (1967), and provided new evidence for within-subjects conditioning effects of flavor novelty in aversion learning.

\section{REFERENCES}

Ahle rs, R. H., \& Best, P. J. Novelty vs. temporal contiguity in learned taste aversions. Psychonomic Science, 1971, 25, 34-35.

Braveman, N. S., \& Jarvis, P. S. Independence of neophobia and taste aversion learning. Animal Learning \& Behavior, 1978, 6, 406-412.

Elkins, R. L. Attenuation of drug-induced bait shyness to a potable solution as an increasing function of its availability prior to conditioning. Behavioral Biology, 1973, 9, 221-226.

Fenwick, S., Mikulka, P. J., \& Klein, S. B. The effects of different levels of preexposure to sucrose on the acquisition and extinction of a conditioned aversion. Behavioral Biology, 1975, 14, 231-235.

Grice, G. R. Stimulus intensity and response evocation. Psychological Review, 1968, 75, 359-373.

KalAt, J. W. Taste salience depends on novelty, not concentration, in taste aversion learning in the rat. Journal of Comparative and Physiological Psychology, 1974, 86, 47-50.

Kalat, J. W., \& Rozin, P. Salience: A factor which can override temporal contiguity in taste aversion learning. Journal of Comparative and Physiological Psychology, 1970, 71, 192-197.

Revusky, S. H., \& BEDARF, E. W. Association of illness with prior ingestion of novel food. Science, 1967, 155, 219-220. 